\title{
Use of polyanions for alkylation of hydrazine derivatives.
}

\author{
Aleksei Bredihhin, Uno Mäeorg* \\ ${ }^{\dagger}$ Institute of Organic and Bioorganic Chemistry, University of Tartu, Jakobi 2, 51014, Tartu, Estonia \\ uno@chem.ut.ee
}

\section{Supporting material}

All reagents were obtained from commercial sources and used without further purification. THF was freshly distilled from $\mathrm{Na} / \mathrm{benzophenone.}$

NMR spectroscopy was performed on a Bruker Avance II 200200 MHz spectrometer using TMS as internal standard. Infrared spectra were measured on a Perkin-Elmer PC16 FTIR spectrometer, using KBr pellet technique for solid compounds and liquid film technique for oils. Melting points were determined on a Gallenkamp melting point apparatus. Compounds $\mathbf{2 b , c}(\mathbf{3} \mathbf{a}, \mathbf{b})$ and $\mathbf{2 d}$ are crystalline solids, others are oils.

\section{Procedures:}

\section{Alkylation of trianion with 3 equiv of alkyl halide (1a); Typical procedure}

An oven-dried flask was charged with BocNHNH 2 (2 mmol, $264 \mathrm{mg})$, then evacuated and backfilled with argon. Thereafter THF $(14 \mathrm{~mL})$ was added to dissolve the solid. Reaction mixture was cooled down to $-78{ }^{\circ} \mathrm{C}$ and $1.6 \mathrm{M} \mathrm{n}-\mathrm{BuLi}$ (6.6 mmol, $4.13 \mathrm{~mL}$ ) solution was added drop wise. Then reaction mixture was allowed to warm up to $-40{ }^{\circ} \mathrm{C}$ for another $30 \mathrm{~min}$ and $\mathrm{Mel}(8 \mathrm{mmol}, 0.5 \mathrm{~mL})$ was added. Then reaction mixture was allowed to slowly warm up to room temperature. The reaction progress was monitored by TLC (Hexane:Et $\mathrm{O}_{2} \mathrm{O}$ 1:1). After 6 hours reaction was mainly complete, but it was allowed to stir overnight. Then volatiles were evaporated (ca. $150 \mathrm{~mm} \mathrm{Hg}, 40{ }^{\circ} \mathrm{C}$ ). To the resulting mixture $15 \mathrm{~mL}$ of DCM and $6 \mathrm{~mL}$ of brine were added. Organic fraction was separated and dried with $\mathrm{MgSO}_{4}$. Then volatiles were removed (ca. $150 \mathrm{~mm} \mathrm{Hg}, 40{ }^{\circ} \mathrm{C}$ ) and residue was purified by column chromatography on silica (Hexane: $\mathrm{Et}_{2} \mathrm{O} 2: 1$ ). 310 mg of colourless oil. Yield was $89 \%$. 


\section{Alkylation of trianion with $\mathbf{2}$ equiv of alkyl halide (2a); Typical procedure}

An oven-dried flask was charged with BocNHNH 2 (2 mmol, $264 \mathrm{mg})$, then evacuated and backfilled with argon. Thereafter THF (14 mL) was added to dissolve the solid. Reaction mixture was cooled down to $-78{ }^{\circ} \mathrm{C}$ and $1.6 \mathrm{M} \mathrm{n}$-BuLi $(6 \mathrm{mmol}, 3.75 \mathrm{~mL}$ ) solution was added drop wise. Then reaction mixture was stirred for $15 \mathrm{~min}$ and Mel (4 mmol, $0.25 \mathrm{~mL}$ ) was added. The reaction progress was monitored by TLC (Hexane: $\mathrm{Et}_{2} \mathrm{O}$ 1:1). Then reaction mixture was stirred for $1 \mathrm{~h}$, then allowed to warm up to room temperature and stirred overnight. Then $0.1 \mathrm{~mL} \mathrm{H}_{2} \mathrm{O}$ was added and volatiles were evaporated. To the resulting mixture $10 \mathrm{~mL}$ of $\mathrm{CHCl}_{3}$ and $\mathrm{MgSO}_{4}$ was added. Then mixture was filtered and volatiles were removed. Residue was purified by column chromatography on silica (Hexane: $\mathrm{Et}_{2} \mathrm{O}$ 1:1). $237 \mathrm{mg}$ of colourless oil obtained. Yield was $74 \%$.

\section{Alkylation of dianion with 2 equiv of alkyl halide (3a); Typical procedure}

An oven-dried flask was charged with $\mathrm{BocNHNH}_{2}(2 \mathrm{mmol}, 264 \mathrm{mg})$, then evacuated and backfilled with argon. Thereafter THF $(10 \mathrm{~mL})$ was added to dissolve the solid. Reaction mixture was cooled down to $-90^{\circ} \mathrm{C}$ and $1.6 \mathrm{M} \mathrm{n}$-BuLi $(4 \mathrm{mmol}, 2.5 \mathrm{~mL})$ solution was added drop wise. Then reaction mixture was stirred for $15 \mathrm{~min}$ and $\mathrm{AllBr}(4 \mathrm{mmol}$, $0.35 \mathrm{~mL}$ ) was added. Then reaction mixture was stirred for $1 \mathrm{~h}$, then allowed to warm up to room temperature and stirred for another $1 \mathrm{~h}$. The reaction progress was monitored by TLC (Hexane: $\mathrm{Et}_{2} \mathrm{O}$ 1:1). Then $0.1 \mathrm{~mL} \mathrm{H}_{2} \mathrm{O}$ was added and volatiles were evaporated. To the resulting mixture $10 \mathrm{~mL}$ of $\mathrm{CHCl}_{3}$ and $\mathrm{MgSO}_{4}$ was added. Then mixture was filtered and volatiles were removed. The residue was purified by column chromatography on silica (Hexane:EtOAc 4:1). $284 \mathrm{mg}$ of colourless crystals obtained. Yield was $67 \%$.

\section{Alkylation of dianion with 1 equiv of alkyl halide (4a); Typical procedure}

An oven-dried flask was charged with BocNHNH 2 (2 mmol, $264 \mathrm{mg})$, then evacuated and backfilled with argon. Thereafter THF $(5 \mathrm{~mL})$ was added to dissolve the solid. Reaction mixture was cooled down to $-90^{\circ} \mathrm{C}$ and $1.6 \mathrm{M} \mathrm{n}$-BuLi ( $4 \mathrm{mmol}, 2.5 \mathrm{~mL}$ ) solution was added drop wise. Then reaction mixture was allowed to warm up to $-50{ }^{\circ} \mathrm{C}$ for 20 min and $\mathrm{AllBr}(1 \mathrm{mmol}, 0.09 \mathrm{~mL})$ was added. Then reaction mixture was stirred for $1 \mathrm{~h}$. The reaction progress was monitored by TLC (Hexane:EtOAc 4:1). Starting material and some amount of tert-butyl-2,2-diallylhydrazinecarboxylate was noticed. Then 
another 0.5 equiv of $\mathrm{AllBr}(1 \mathrm{mmol}, 0.09 \mathrm{~mL})$ was added. After $1 \mathrm{~h} 0.2 \mathrm{~mL}$ of $\mathrm{MeOH}$ was added followed by $0.2, \mathrm{~mL} \mathrm{H}_{2} \mathrm{O}$. After that reaction mixture was allowed to warm up to room temperature. The reaction mixture was evaporated. To the resulting mixture 15 $\mathrm{mL}$ of $\mathrm{DCM}$ and $6 \mathrm{~mL}$ of brine were added. Organic fraction was separated and dried with $\mathrm{MgSO}_{4}$. Then volatiles were removed and residue was purified by column chromatography on silica (Hexane:EtOAc 4:1). $175 \mathrm{mg}$ of colourless oil obtained. Yield was $50 \%$.

\section{Analytical data:}

tert-butyl 1,2,2-trimethylhydrazinecarboxylate (1a)

${ }^{1} \mathrm{H}$ NMR (200 MHz, $\left.\mathrm{CDCl}_{3}, \mathrm{TMS}\right): \delta=2.91(\mathrm{~s}, 6 \mathrm{H}), 2.61(\mathrm{~s}, 3 \mathrm{H}), 1.48(\mathrm{~s}, 9 \mathrm{H})$.

${ }^{13} \mathrm{C}$ NMR (100 MHz, $\left.\mathrm{CDCl}_{3}, \mathrm{TMS}\right): \delta=155.6,79.9,42.7,33.0,28.7$.

FT IR $v\left(\mathrm{~cm}^{-1}\right):$ 2972, 2952, 2885, 1698, 1487, 1452, 1364, 1154, 768.

\section{tert-butyl 1,2,2-triallylhydrazinecarboxylate (1b)}

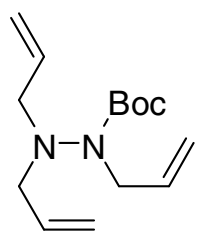

${ }^{1} \mathrm{H}$ NMR (200 MHz, $\left.\mathrm{CDCl}_{3}, \mathrm{TMS}\right): \delta=5.94-5.74(\mathrm{~m}, 3 \mathrm{H}), 5.22-5.05(\mathrm{~m}, 6 \mathrm{H}), 3.84$ (broad s, 3H), 3.47 (broad s, 3H), 1.47 (s, 9H).

${ }^{13} \mathrm{C} \mathrm{NMR}\left(100 \mathrm{MHz}, \mathrm{CDCl}_{3}, \mathrm{TMS}\right): \delta=155.1,135.8,135.2,117.3,116.4,80.1,58.1$, 55.1, 28.7.

FT IR $v\left(\mathrm{~cm}^{-1}\right):$ 3080, 2977, 2930, 2859, 1693, 1374, 1246, 1179, 1130, 994, 927, 763. HRMS (ESI): $\mathrm{m} / \mathrm{z}$ calcd for $\mathrm{C}_{14} \mathrm{H}_{24} \mathrm{~N}_{2} \mathrm{O}_{2}[\mathrm{MH}]^{+}:$253.19105; found:253.19110.

\section{tert-butyl 2,2-dimethylhydrazinecarboxylate (2a)}<smiles>CO[R16](=O)N([AlH])N(C)C</smiles>

${ }^{1} \mathrm{H}$ NMR (200 MHz, CDCl 3 , TMS): $\delta=5.65$ (broad s, 1H), $2.58(\mathrm{~s}, 6 \mathrm{H}), 1.46(\mathrm{~s}, 9 \mathrm{H})$.

${ }^{13} \mathrm{C}$ NMR (100 MHz, $\left.\mathrm{CDCl}_{3}, \mathrm{TMS}\right): \delta=154.7,80.0,47.6,28.4$.

FT IR $v\left(\mathrm{~cm}^{-1}\right):$ 3244, 2982, 2864, 2787, 1708, 1518, 1451, 1251, 1159, 1107, 846.

tert-butyl 2,2-diallylhydrazinecarboxylate (2b)

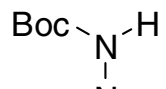

${ }^{1} \mathrm{H}$ NMR (200 MHz, $\left.\mathrm{CDCl}_{3}, \mathrm{TMS}\right): \delta=6.01-5.81(\mathrm{~m}, 2 \mathrm{H}), 5.53$ (broad s, $1 \mathrm{H}$ ), 5.26-5.15 $(\mathrm{m}, 6 \mathrm{H}), 3.42 / 3.39(\mathrm{~s}, 4 \mathrm{H}), 1.43(\mathrm{~s}, 9 \mathrm{H})$,

${ }^{13} \mathrm{C}$ NMR (100 MHz, $\left.\mathrm{CDCl}_{3}, \mathrm{TMS}\right): \delta=155.3,134.2,118.4,79.7,59.8,28.4$. 
FT IR $v\left(\mathrm{~cm}^{-1}\right): 3249,3080,2982,2920,2859,1703,1503,1359,1241,1164,995,907$, $845,742,614$.

HRMS (ESI): $\mathrm{m} / \mathrm{z}$ calcd for $\mathrm{C}_{11} \mathrm{H}_{20} \mathrm{~N}_{2} \mathrm{O}_{2}[\mathrm{MH}]^{+}:$:213.15975; found:.213.15965 $\mathrm{mp} 38-40{ }^{\circ} \mathrm{C}$

tert-butyl 2,2-dibenzylhydrazinecarboxylate (2c)

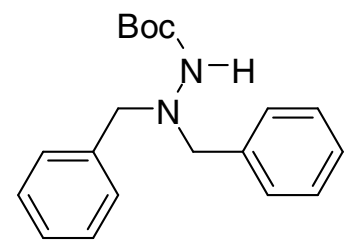

${ }^{1} \mathrm{H}$ NMR (200 MHz, $\left.\mathrm{CDCl}_{3}, \mathrm{TMS}\right): \delta=7.40-7.24(\mathrm{~m}, 10 \mathrm{H}), 5.68$ (broad s, $\left.1 \mathrm{H}\right) 4.05$ (broad s, 2H), 1.35 (s, 9H).

${ }^{13} \mathrm{C} \mathrm{NMR}\left(100 \mathrm{MHz}, \mathrm{CDCl}_{3}, \mathrm{TMS}\right): \delta=155.2,137.7,129.3,128.3,127.3,79.8,60.4$, 28.4.

FT IR $v\left(\mathrm{~cm}^{-1}\right): 3305,3090,3064,3034,2977,2936,2869,2828,1693,1508,1456$, $1364,1266,1236,1153,742,691$.

$\mathrm{mp} 115-118^{\circ} \mathrm{C}$

tert-butyl pyrrolidin-1-ylcarbamate (2d)

${ }^{1} \mathrm{H}$ NMR $\left(200 \mathrm{MHz}, \mathrm{CDCl}_{3}, 2{ }^{\circ} \mathrm{C}, \mathrm{TMS}\right): \delta=5.62(\mathrm{~s}$ broad, $1 \mathrm{H}), 2.87(\mathrm{~m}, 4 \mathrm{H}), 1.82(\mathrm{~m}, 4$ $\mathrm{H}), 1.46(\mathrm{~s}, 9 \mathrm{H})$.

${ }^{13} \mathrm{C}$ NMR (100 MHz, $\left.\mathrm{CDCl}_{3}, 2{ }^{\circ} \mathrm{C}, \mathrm{TMS}\right): \delta=155.0,79.9,55.1,28.5,22.4$.

FT IR $v\left(\mathrm{~cm}^{-1}\right):$ 3229, 2977, 2925, 2843, 1688, 1544, 1282, 1359, 1164, 860, 624.

HRMS (ESI): $\mathrm{m} / \mathrm{z}$ calcd for $\mathrm{C}_{9} \mathrm{H}_{18} \mathrm{~N}_{2} \mathrm{O}_{2}[M H]^{+}:$187.14410; found:.187.14421

mp $110-114{ }^{\circ} \mathrm{C}$

tert-butyl 2-allylhydrazinecarboxylate (4a)

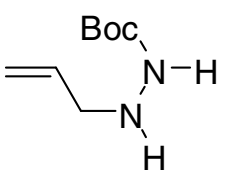

${ }^{1} \mathrm{H}$ NMR $\left(200 \mathrm{MHz}, \mathrm{CDCl}_{3}, 2{ }^{\circ} \mathrm{C}, \mathrm{TMS}\right): \delta=6.71 / 6.47$ (broad s, $\left.1 \mathrm{H}\right), 5.98-5.76(\mathrm{~m}, 2 \mathrm{H})$, 5.26-5.12 (m, 2H), $3.97(\mathrm{~s}, 1 \mathrm{H}), 3.48 / 3.45(\mathrm{~s}, 2 \mathrm{H}), 1.46(\mathrm{~s}, 9 \mathrm{H})$,

${ }^{13} \mathrm{C}$ NMR (100 MHz, $\left.\mathrm{CDCl}_{3}, 21{ }^{\circ} \mathrm{C}, \mathrm{TMS}\right): \delta=156.8,134.5,117.9,80.3,54.6,28.4$.

FT IR $v\left(\mathrm{~cm}^{-1}\right): 3311,3075,2972,2930,2864,1703,1456,1380,1282,1251,1158$, 927. 


\section{tert-butyl 1,2,2-trimethylhydrazinecarboxylate (1a)}

$$
\text { in }
$$
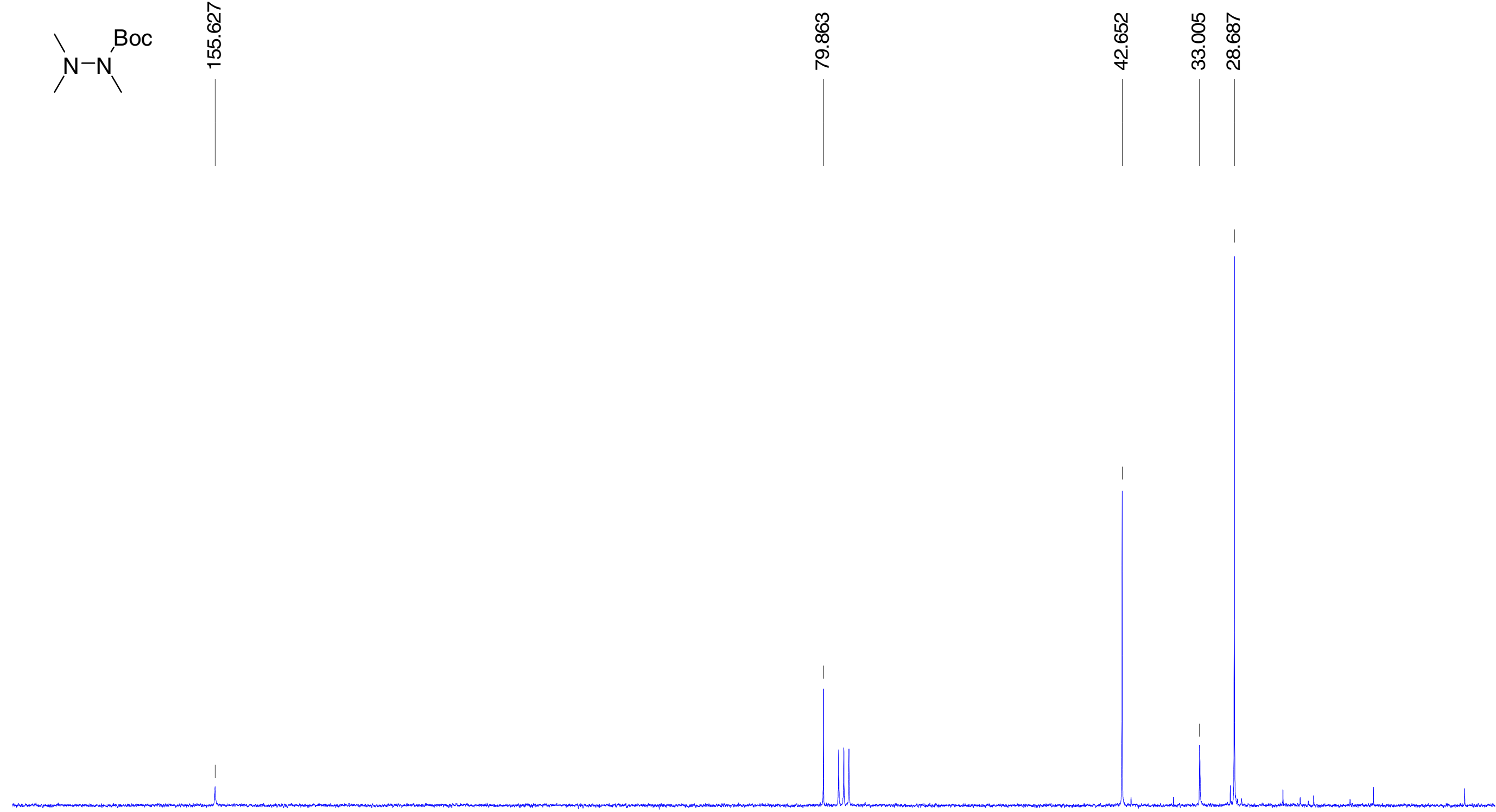


\section{tert-butyl 1,2,2-triallylhydrazinecarboxylate (1b)}

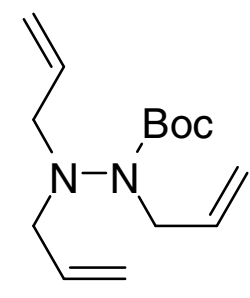

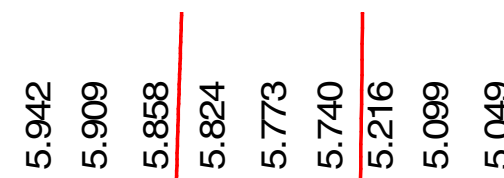

\begin{tabular}{l|l} 
& \\
0 & $\infty$ \\
$\infty$ & 0 \\
$\infty$ & 0 \\
$\infty$ & 0
\end{tabular}

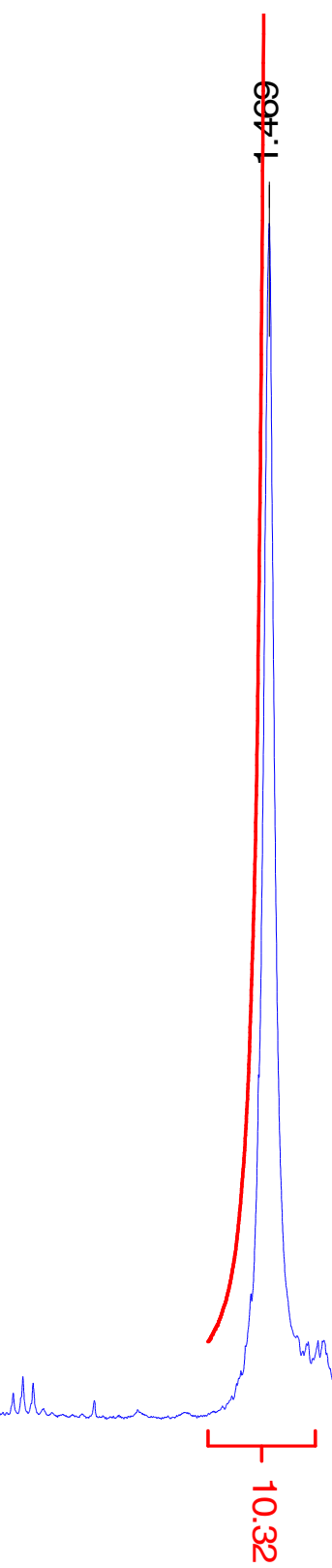

9.0

8.0

7.0

6.0

5.0

4.0

3.0

2.0

1.0

0.0 


\section{tert-butyl 1,2,2-triallylhydrazinecarboxylate (1b)}
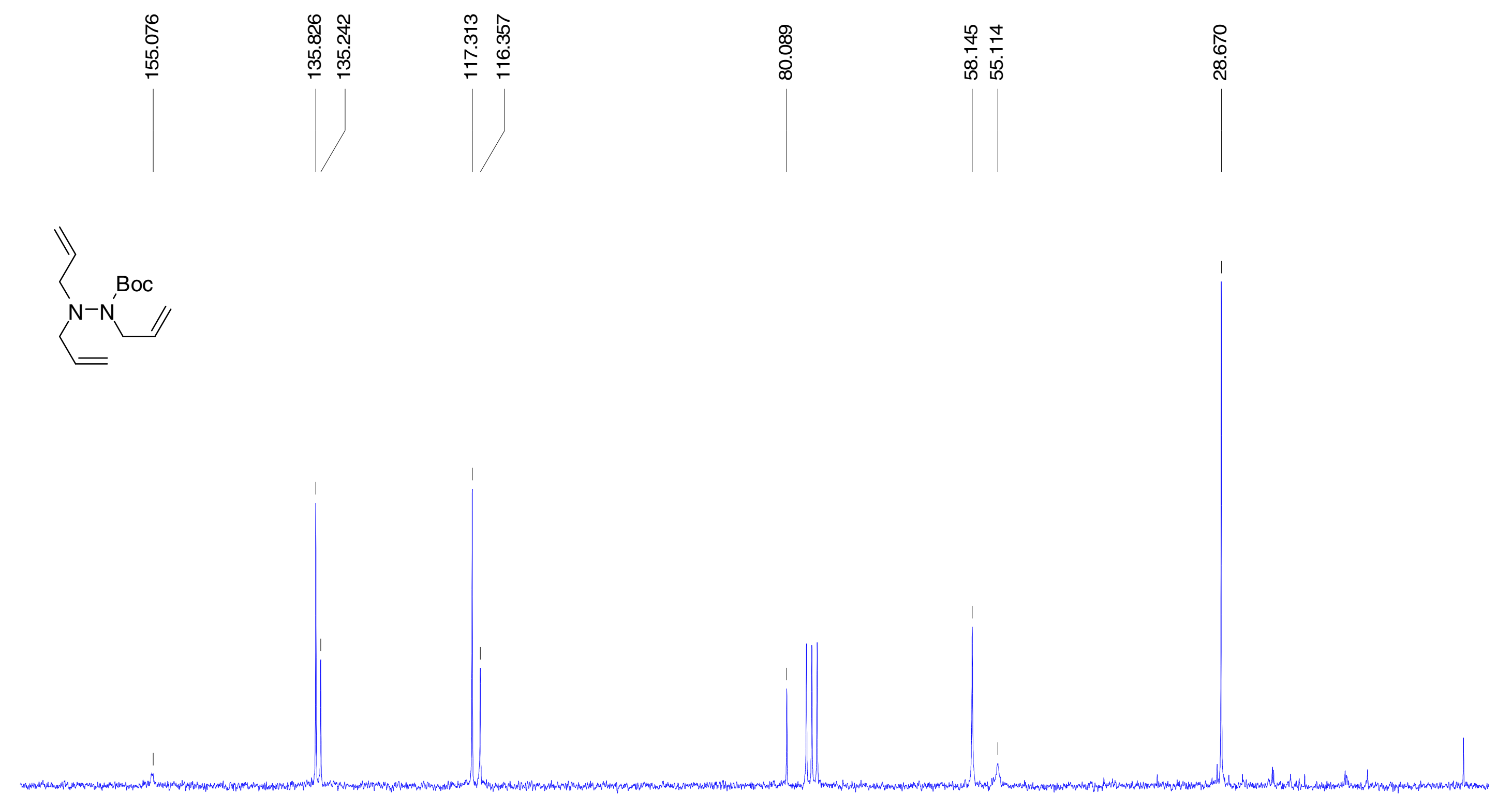
tert-butyl 2,2-dimethylhydrazinecarboxylate (2a)
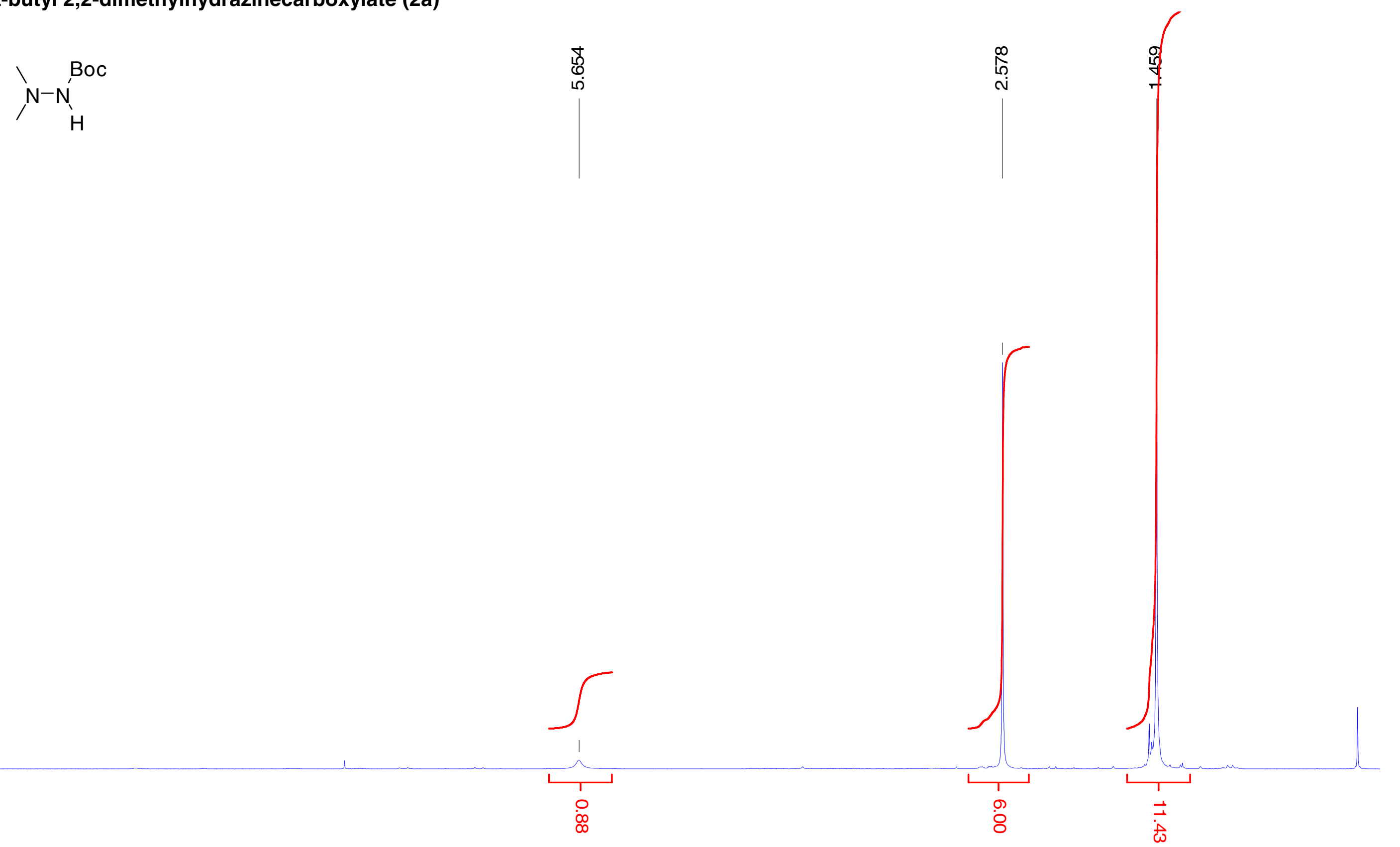

$\infty$

8 
tert-butyl 2,2-dimethylhydrazinecarboxylate (2a)

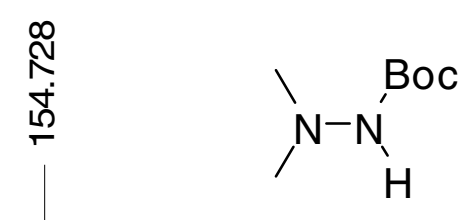




\section{tert-butyl 2,2-diallylhydrazinecarboxylate (2b)}
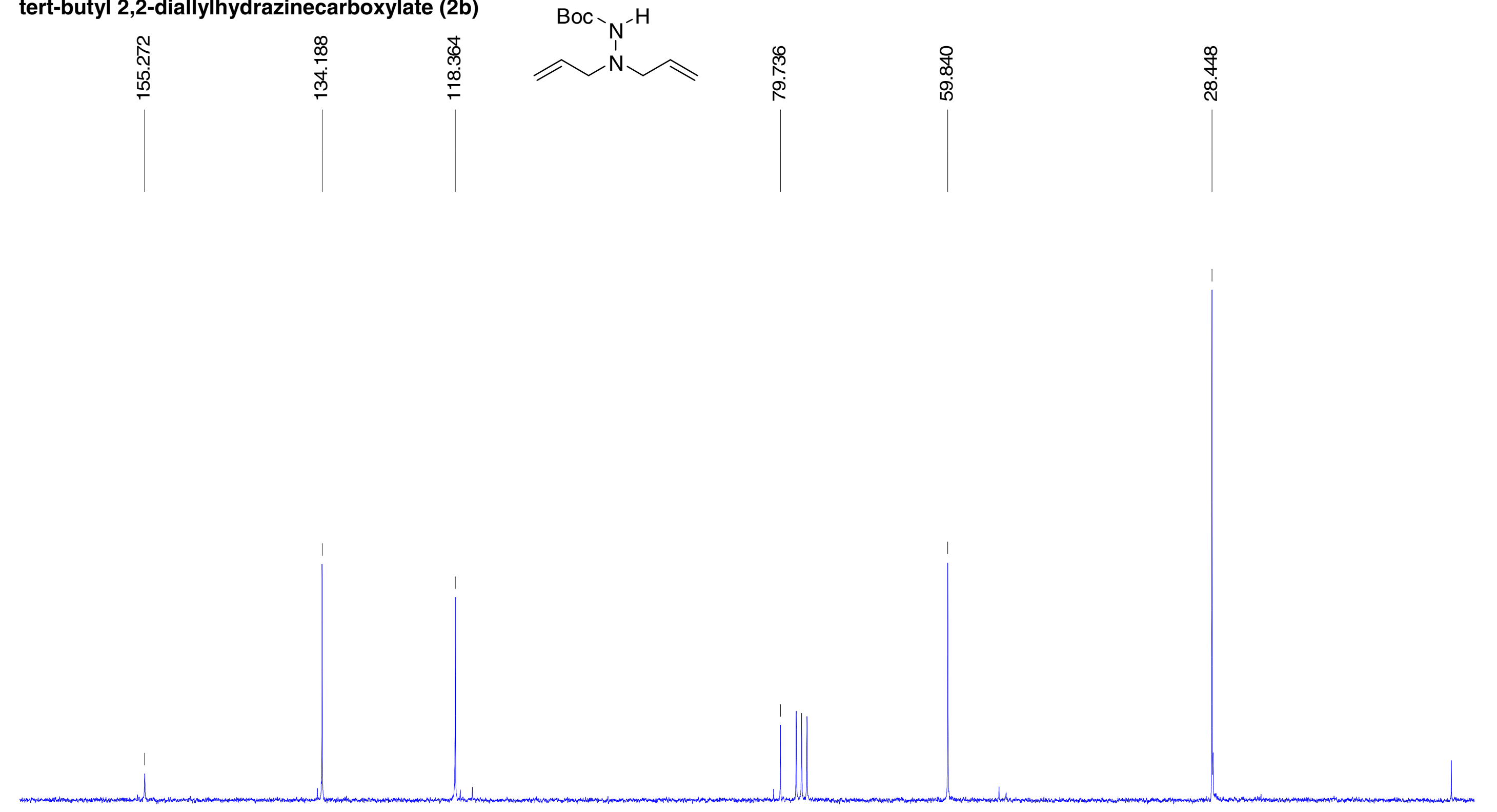


\section{tert-butyl 2,2-dibenzylhydrazinecarboxylate (2c)}
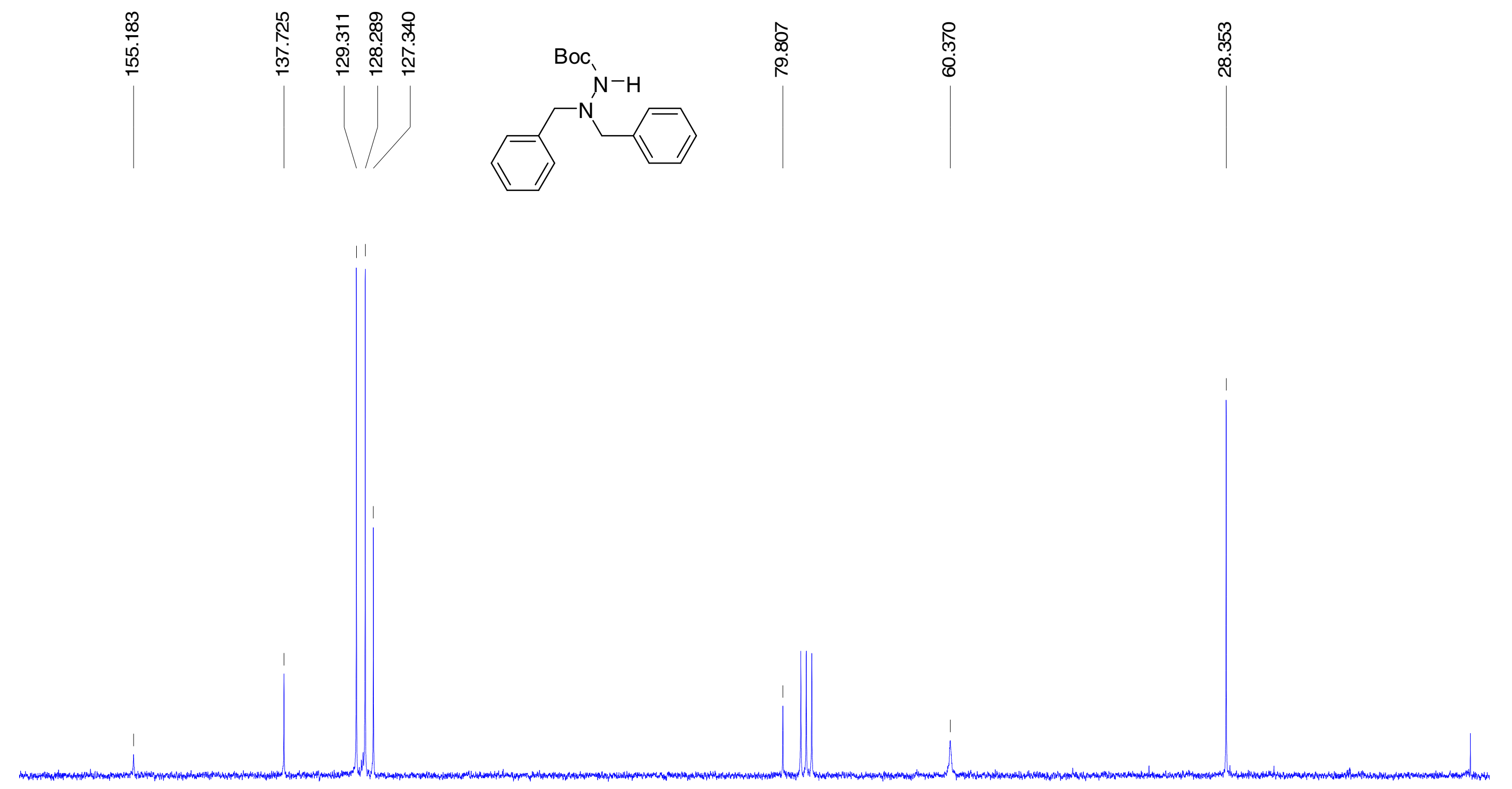


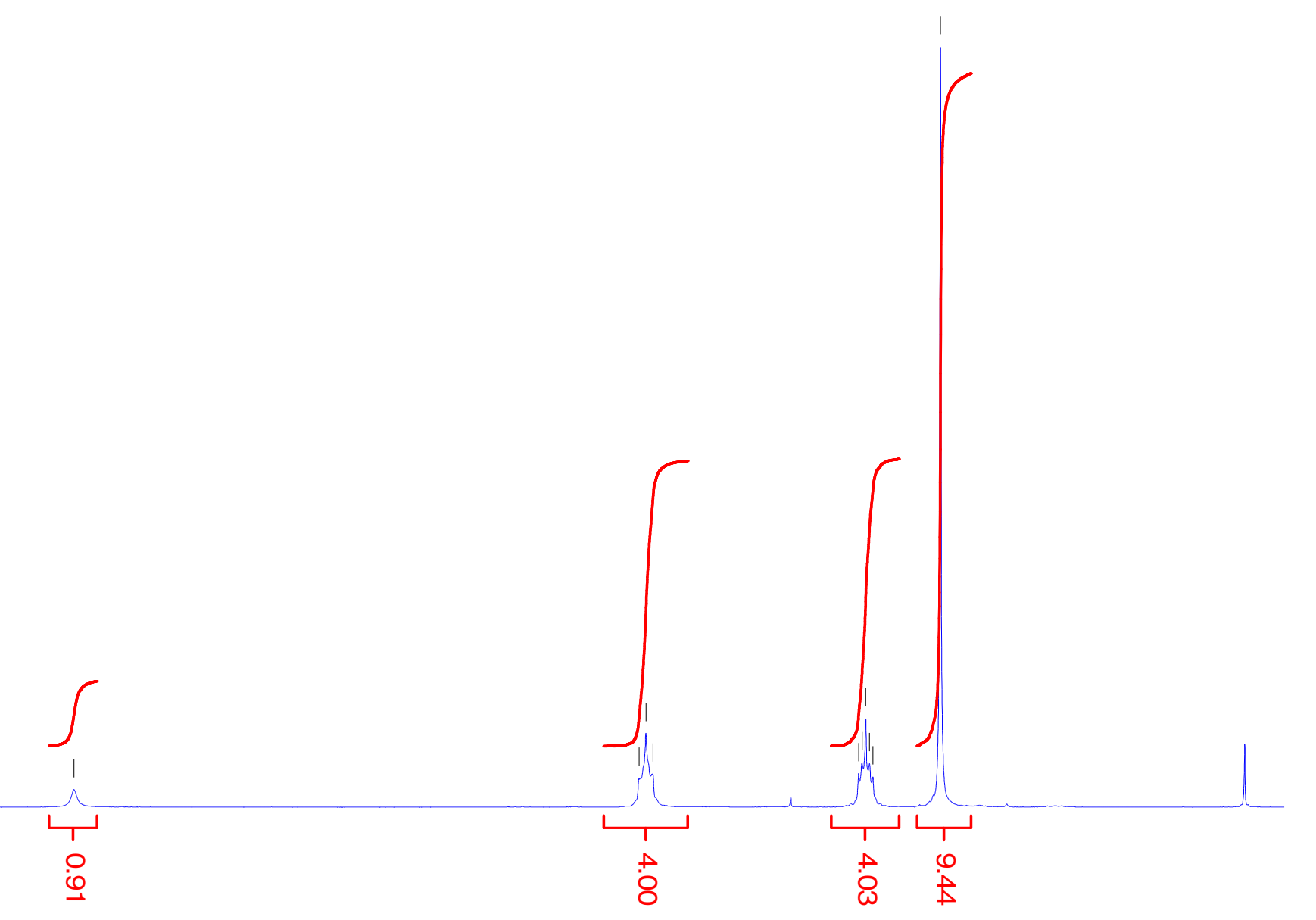




\section{tert-butyl pyrrolidin-1-ylcarbamate (2d)}
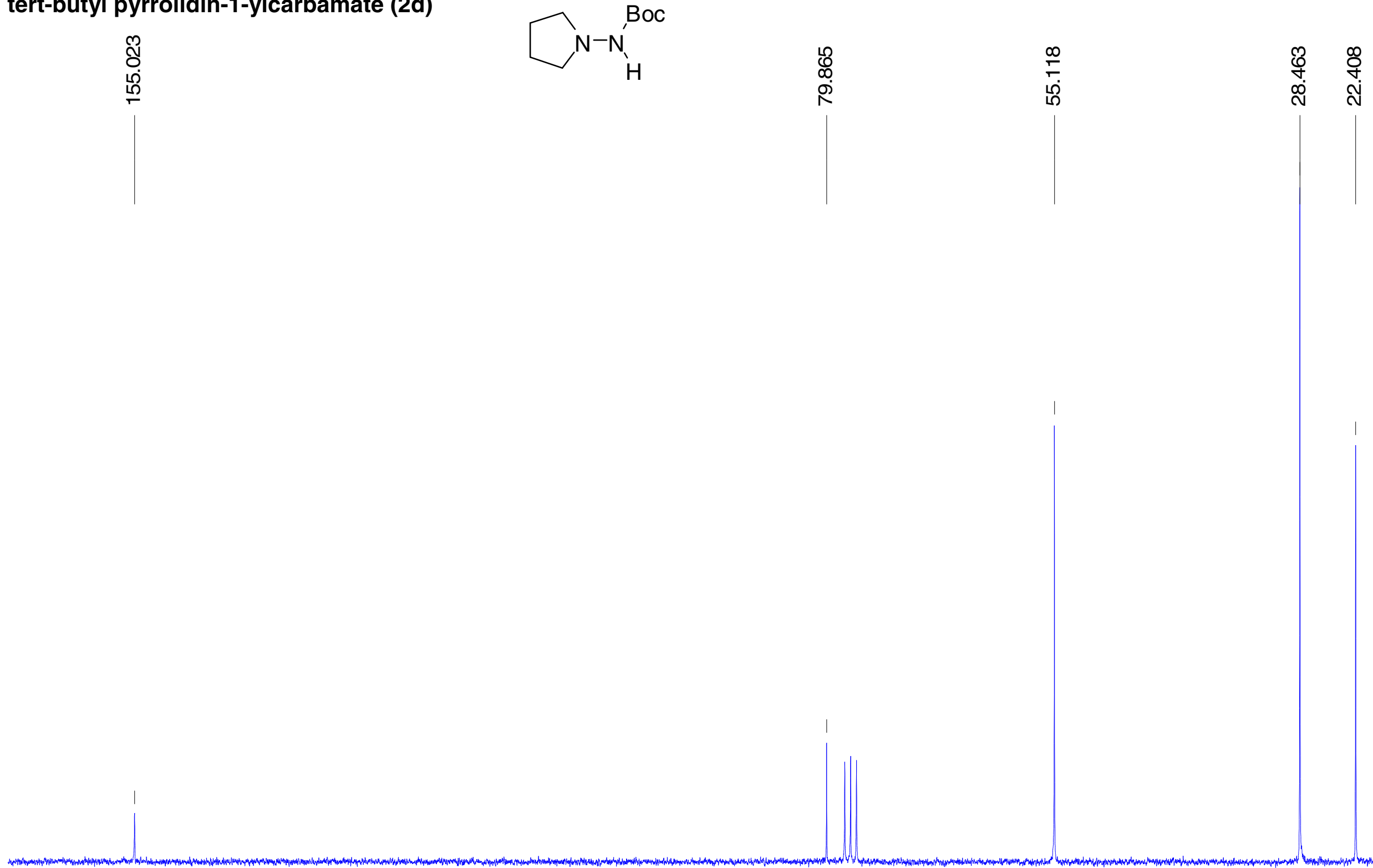
tert-butyl 2-allylhydrazinecarboxylate (3a)

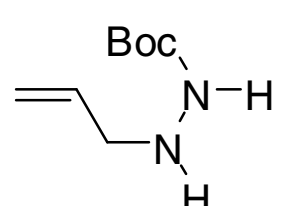

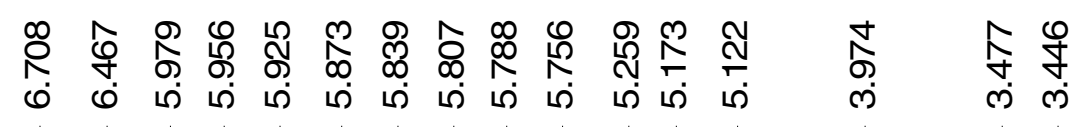
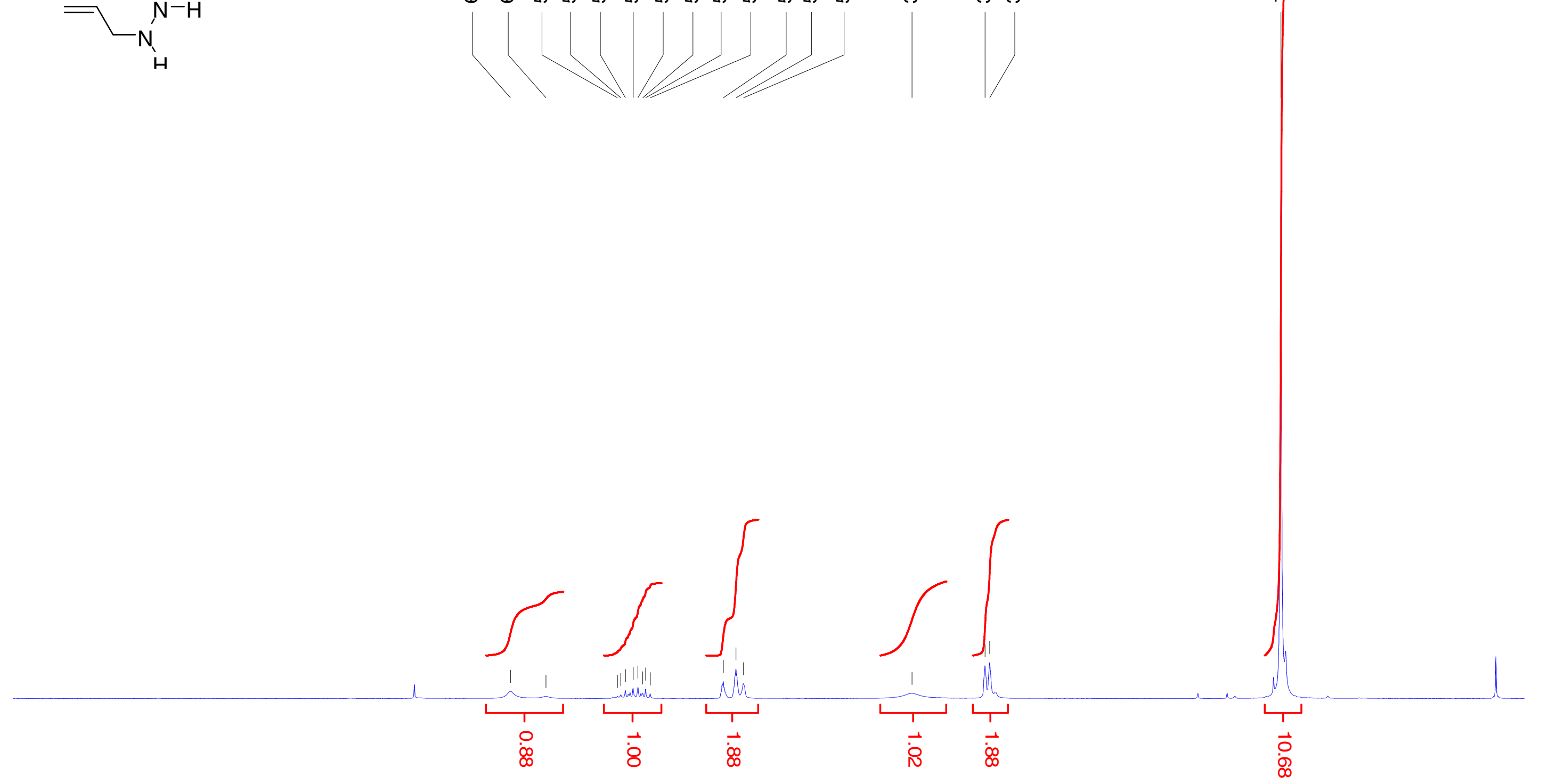


\section{tert-butyl 2-allylhydrazinecarboxylate (3a)}

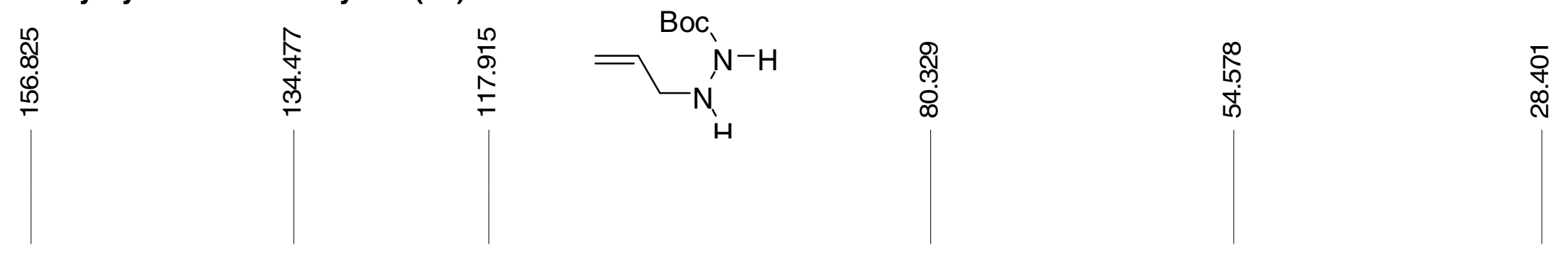

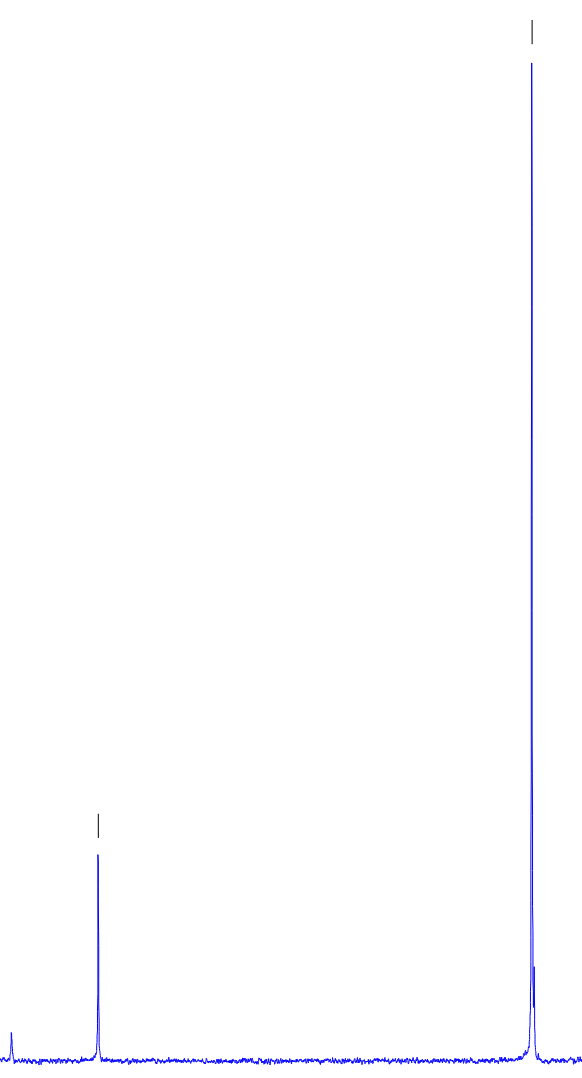

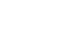

